\title{
THE THEORETICAL RESEARCH OF AN UPWARD TWO-PHASE FLOW WITH PHASE'S CHANGES IN A VERTICAL WELL
}

\author{
Shagapov V.Sh. ${ }^{1}$, Borodin S.L. ${ }^{2}$, Gubaidullin A.A. ${ }^{2}$, Duong Ngoc Hai ${ }^{3}$, \\ Musakaev N.G. ${ }^{2}$ \\ ${ }^{1}$ Birsk State Social-and-Pedagogical Academy, Russia, \\ ${ }^{2}$ Tyumen Branch of Khristianovich Institute of Theoretical and \\ Applied Mechanics SB RAS, Russia, \\ ${ }^{3}$ Institute of Mechanics, VAST, Vietnam.
}

\begin{abstract}
By methods of mechanics of multiphase systems the mathematical model describing an upward gas-and-oil flow in a vertical well is constructed. The model considers phase's changes, deposition of oil paraffin on the elevating column's walls of the well, influence of an installation of electrical centrifugal pumps on parameters of the gas-and-oil flow, and also heat exchange of the well with surrounding rock.

It is shown, that at the using of an installation of electrical centrifugal pumps and variation of its metering characteristic, depth of its setting, it is possible to achieve the optimum temperature mode on all the well's height and to prevent by it formation of paraffin deposits.
\end{abstract}

\section{INTRODUCTION}

Many oil fields are characterized by high content in oil of heavy high-molecular hydrocarbons (oil paraffin). Movement of such oil from reservoirs to oil-gathering systems is complicated by processes of formation in pipes hard paraffin deposits. Primary reason of paraffin's deposition is reduction of temperature in a well which leads to reduction of oil's dissolving ability. Therefore at research of an upward gas-and-oil flow in a vertical well it is necessary to take into account three interconnected factors: hydrodynamics and thermal physics of the gas-liquid flow in the well, growth of paraffin deposits on the inner wall of the well's elevating column, thermal interaction of the gas-liquid flow in the well with surrounding rock. If the well are being exploiting by an installation of electrical centrifugal pumps (IECP), then also it is necessary to take into account jumping of flow's parameters owing to the IECP work.

For the theoretical research of features of an upward gas-and-oil flow in a vertical well, it is necessary to construct the mathematical model, which would take into account all above-named factors. 


\section{THE MATHEMATICAL MODEL}

Let's consider an axial-symmetric flow of a gas-and-oil mixture in the vertical well in radius $R_{0}$, depth $H$. The well is equipped by the IECP of length $L$. Let's direct the axis $Z$ vertically upwards, with it's origin at the well bottom.

Let's accept that the gas-liquid mixture in the well consists of three components: the heavy $(\mathrm{H})$, the medium $(\mathrm{M})$ and the light $(\mathrm{L})$. At the liquid phase there are all three components; at the gas phase there are the medium and the light components [1].

At the mathematical modeling of the gas-liquid flow for the basis let's accept following assumptions: the temperature for each section of the channel is equal for the both phases (gas and liquid); phase's transitions are occurring in equilibrium mode; the flow in the well bore is quasisteady.

The conservation of mass equations for the both phases let's write in the form [2]:

$$
\frac{d m_{l}}{d z}=-J^{l g}-J^{l s}, \quad \frac{d m_{g}}{d z}=J^{l g},
$$

where $m_{i}(i=l, g)$ is the mass flow of the $i$ th phase through the section of the well with the co-ordinate $z ; J^{l g}$ and $J^{l s}$ are, accordingly, the intensities of gas release and release of paraffin deposits, carried to the unit of channel's length. Here and further the bottom indexes $l$ and $g$ are corresponding to parameters of the liquid and gas phases; the bottom indexes in brackets are corresponding to parameters of the heavy, the medium and the light components.

If to neglect mass changing of the gas-liquid mixture in the well because of deposition of the paraffin $\left(J^{l s} \approx 0\right)$, then the equation (1) can be written in the form:

$$
m_{l}+m_{g}=m=\text { const, }
$$

The equation (2) means that the full mass flow does not vary throughout the well height.

The momentum equations for the liquid and gas phases in stationary approach can be written in the form:

$$
\begin{gathered}
\frac{d\left(m_{l} v_{l}\right)}{d z}=-S_{l} \frac{d p}{d z}-f-f_{l w}-J^{l g} v_{g l}-J^{l s} v_{l s}-\rho_{l}^{0}(1-\alpha) S g, \\
\frac{d\left(m_{g} v_{g}\right)}{d z}=-S_{g} \frac{d p}{d z}+f-f_{g w}+J^{l g} v_{l g}-\rho_{g}^{0} \alpha S g
\end{gathered}
$$

where $v_{i}$ and $\rho_{i}^{0}(i=l, g)$ are, accordingly, the velocity and the real density of the $i$ th phase; $f$ - the power of interphase interaction; $f_{i w}$ - the friction between the $i$ th phase and the wall of the channel; $g$ - gravitational acceleration; $\alpha$ - the volumetric gas content; $\delta_{s}$ - the thickness of the paraffin deposits on the elevating column's inner wall; $J^{l g} v_{l g}$ and $J^{l s} v_{l s}$ are connected with the impulse's transfer because of phase transitions.

Let's transform the equations (3) and (4) taking into account the conservation of mass equations (1) and then having combined them, we will receive the momentum equation for the all mixture:

$$
m_{l} \frac{d v_{l}}{d z}+m_{g} \frac{d v_{g}}{d z}=-S \frac{d p}{d z}-f_{w}+J^{l g}\left(v_{l}-v_{g}\right)-J^{l s}\left(v_{l s}-v_{l}\right)-\rho S g
$$


where $f_{w}=f_{l w}+f_{g w}, \rho=\rho_{l}^{0}(1-\alpha)+\rho_{g}^{0} \alpha, S=S_{l}+S_{g}, S=\pi R^{2}, R=R_{0}-\delta_{s}, \delta_{s}$ is the thickness of the paraffin deposits on the inner wall of the well's elevating column.

As speed of the gas-liquid flows in the wells is much less then acoustic speed in corresponding mediums, probably to neglect by components in the left part of the equation (5), which connected with accelerative effects. Besides it is possible to neglect also by the reaction powers $J^{l s}\left(v_{l s}-v_{l}\right)$ and $J^{l g}\left(v_{l}-v_{g}\right)$, which connected with the phase transitions. In that case the equation (5) can be written in the form:

$$
\begin{aligned}
& \frac{d p}{d z}=-F_{w}-\rho g \\
& F_{w}=\frac{\lambda_{w} \rho W^{2}}{2 R}, \quad\left[\lambda_{w}=\lambda_{0} \psi\right]
\end{aligned}
$$

where $W=W_{l}+W_{g}, W_{i}(i=l, g)$ is the specific velocity of the $i$ th phase.

For homogeneous flow's resistance coefficient $\lambda_{0}$ let's accept Churchill's equation [3], which valid in all range of Reynolds numbers:

$$
\begin{aligned}
& \lambda_{0}=8\left[(8 / \mathrm{Re})^{12}+\left(A_{0}+B_{0}\right)^{-3 / 2}\right]^{1 / 12}, \\
& A_{0}=\left[-2,457 \ln \left((7 / \operatorname{Re})^{0,9}+0,135 \varepsilon / \kappa\right)\right]^{16}, \quad B_{0}=(37530 / \operatorname{Re})^{16}, \\
& \operatorname{Re}=2 R \rho W / \mu, \quad \mu=x \mu_{g}+(1-x) \mu_{l}, \quad x=m_{g} /\left(m_{l}+m_{g}\right),
\end{aligned}
$$

where $\varepsilon$ is the size of roughness of the inner surface of the pipes; $\mu_{i}(i=l, g)$ - the dynamic viscosity of the $i$ th phase.

The correction $\psi$ for the vertical upward two-phase flow at bubble flow is accepted equal to one, at piston flow:

$$
\begin{gathered}
\psi=\frac{1}{1-\varphi}\left\{1-0,78 \varphi[1-\exp (-2,2 \sqrt{F r})]-0,22 \varphi\left[1-\exp \left(1-\frac{15 \rho_{l}^{0}}{\rho_{g}^{0}}\right)\right]\right\}, \\
F r=\frac{\rho_{l}^{0} W^{2}}{2 g \Delta \rho R}, \quad \Delta \rho=\rho_{l}^{0}-\rho_{g}^{0}, \quad \varphi=\left(1+\frac{\rho_{g}^{0} m_{l}}{\rho_{l}^{0} m_{g}}\right)^{-1} .
\end{gathered}
$$

For calculation of the value of the volumetric gas content, usually use the empirical dependences:

$$
\begin{aligned}
& \text { bubble flow }-\alpha=\frac{\varphi}{1,2+1,53 F r^{-0,5}\left(\sigma / 4 g \Delta \rho R^{2}\right)^{0,25}}, \\
& \text { piston flow }-\alpha=\frac{\varphi}{1,2+0,35 F r^{-0,5}},
\end{aligned}
$$

where $\sigma$ is the coefficient of surface tension.

If Froude's number $F r \gg 1$, then it is possible to accept that the $\alpha \approx 0,83 \varphi$.

The equation of changing of the total energy can be written in the form:

$$
\begin{aligned}
\frac{d}{d z}\left[m_{l}\right. & \left.\left(u_{l}+\frac{v_{l}^{2}}{2}\right)+m_{g}\left(u_{g}+\frac{v_{g}^{2}}{2}\right)\right]=-f_{l w} v_{l}-f_{g w} v_{g}- \\
& -\frac{d}{d z}\left[p\left(S_{l} v_{l}+S_{g} v_{g}\right)\right]-\left(\rho_{l}^{0} \alpha_{l} v_{l}+\rho_{g}^{0} \alpha_{g} v_{g}\right) S g-Q_{w},
\end{aligned}
$$


where $u_{i}$ is the specific internal energy of the $i$ th phase; $Q_{w}=2 \pi R q_{w}$ - the heat current intensity per unit length of the well.

The compressibility of the liquid phase we will neglect, i.e. $\rho_{l}^{0}=$ const. For the gas phase we will accept equation of Klajperona-Mendeleeva: $p=\rho_{g}^{0} R_{g} T$.

Let the pressure in the gas phase satisfies to Dalton law:

$$
\begin{aligned}
& p=p_{(M)}+p_{(L)}, \\
& p_{(M)}=\rho_{g(M)}^{0} R_{(M)} T, \quad p_{(L)}=\rho_{g(L)}^{0} R_{(L)} T, \\
& \rho_{g(M)}^{0}+\rho_{g(L)}^{0}=\rho_{g}^{0}, \quad R_{(M)}\left(1-k_{g(L)}\right)+R_{(L)} k_{g(L)}=R_{g},
\end{aligned}
$$

where $k_{i(L)}(i=l, g)$ is the mass concentration of the light component in the $i$ th phase.

Let's assume that the partial pressure of the steams of the liquid (medium component) is equal to the saturation pressure's value at the current temperature and is approximated by the following function:

$$
p_{(M)}=p_{(M)}(T)=p_{*} \exp \left(-T_{*} / T\right),
$$

where $p_{*}, T_{*}$ are the empirical approximating parameters.

For the dependence of the light component's partial pressure from its mass concentration in the liquid phase we will pass Henry's law:

$$
p_{(L)}=G_{(L)} k_{l(L)}, \quad\left(G_{(L)}=p_{(L) 0} / k_{l(L) 0}\right)
$$

$G_{(L)}$ - Henry's constant.

On the basis of the Equations (9) - (11), excluding $p_{(M)}$ and $p_{(L)}$, it is possible to receive:

$$
\begin{aligned}
& p=p_{(M)}(T)+G_{(L)} k_{l(L)}, \\
& \frac{p_{(M)}(T)}{p}=\frac{R_{(M)}\left(1-k_{g(L)}\right)}{R_{(M)}\left(1-k_{g(L)}\right)+R_{(L)} k_{g(L)}}=B .
\end{aligned}
$$

If we differentiate the equation (12) on co-ordinate $z$, then we have:

$$
\begin{gathered}
\frac{d p}{d z}=\frac{d p_{(M)}(T)}{d z}+G_{(L)} \frac{d k_{l(L)}}{d z}, \\
\frac{d p_{(M)}(T)}{d z}=p_{(M)}^{\prime} \frac{d T}{d z}=B \frac{d p}{d z}+B^{\prime} p \frac{d k_{g(L)}}{d z}, \quad p_{(M)}^{\prime}=\frac{d p_{(M)}(T)}{d T}=\frac{T_{*} p_{(M)}(T)}{T^{2}}, \\
\frac{d B}{d k_{g(L)}}=B^{\prime}=-\frac{R_{(M)} R_{(L)}}{\left[R_{(M)}\left(1-k_{g(L)}\right)+R_{(L)} k_{g(L)}\right]^{2}} .
\end{gathered}
$$

Then for the light component's mass concentrations in the liquid and gas phases can be received:

$$
\begin{gathered}
\frac{d k_{l(L)}}{d z}=\left(\frac{d p}{d z}-p_{(M)}^{\prime} \frac{d T}{d z}\right) / G_{(L)}, \\
\frac{d k_{g(L)}}{d z}=\left(p_{(M)}^{\prime} \frac{d T}{d z}-B \frac{d p}{d z}\right) / B^{\prime} p .
\end{gathered}
$$


From the equation (8), using the equations (1), (3), (4), (13) and (14), it is possible to receive:

$$
\begin{aligned}
& \frac{d T}{d z}=\frac{\left(\frac{m_{g}}{\rho_{g}^{0}}+\frac{A_{l}}{G_{(L)}}-\frac{A_{g} \cdot B}{B^{\prime} p}\right) \frac{d p}{d z}-Q_{w}}{m c+\frac{A_{l} p_{(M)}^{\prime}}{G_{(L)}}-\frac{A_{g} p_{(M)}^{\prime}}{B^{\prime} p}}, \\
& A_{l}=m_{l}\left(L_{(L)}-L_{(M)}+L_{(L M)}\right), \quad A_{g}=m_{g} L_{(L M)}, \\
& L_{(L M)}=\frac{L_{(L)} k_{l(L)}+L_{(M)}\left(1-k_{l(L)}\right)}{k_{g(L)}-k_{l(L)}}, \quad m c=m_{l} c_{l}+m_{g} c_{g},
\end{aligned}
$$

where $L_{(M)}$ and $L_{(L)}$ are, accordingly, the specific heat's of the liquid phase's evaporation and releasing of the gas dissolved in the liquid; $c_{i}(i=l, g)$ - the specific heat of the $i$ th phase at constant pressure.

From system of the equations (6), (13) - (15) it is possible to define the change of pressure, the temperature and the components' mass concentrations on the well height. For closing of the given system it is necessary to set the intensity of solid deposits' growth on the internal walls of the elevating column, to specify the changes of pressure and temperature of the liquid-gas flow as the result of IECP work, and also to set the intensity of heat transfer to surrounding rock.

Oil wells usually consist of two coaxial metal pipes, namely: from an internal elevating column, on which the well product flows and an external casing column. The interval between columns is filled by a gas or a liquid.

For heat transfer's coefficient through system of the pipes of the well the following expression is used [4]:

$$
\frac{1}{\beta}=R_{N} \sum_{i=1}^{N} \lambda_{i}^{-1} \ln \left(R_{i} / R_{i-1}\right)+\frac{1}{\beta_{e x t}}, \quad\left(R_{N}=R_{c}\right)
$$

where $R_{i}$ is the external radius of the $i$ th layer, thus $i=1$ is corresponding to the elevating column, $i=N$ is the layer, contiguous to surrounding rock; $\beta_{\text {ext }}$ - heat transfer's coefficient of surrounding rock.

The account of natural convection of a medium in the annular space can be made with the help of the multiplier for thermal conductivity of the medium in the specified volume [4]:

$$
\zeta=0,049(\mathrm{Gr} \cdot \operatorname{Pr})^{1 / 3} \operatorname{Pr}^{0,074},
$$

where Gr and Pr - numbers of Grashof and Prandtl.

To determine the intensity of heat exchange between the flow and the wall of the well (or the solid phase on the wall of the well), let's allocate two sites on the depth of the well. The first site is from the well's bottom to well's section, where on the inner walls of the injection column deposition of the hard phase is started. On this site for the intensity of heat exchange it is possible to write:

$$
q_{w}=\beta_{w}\left(T-T_{w}\right)
$$


where $T_{w}$ is the temperature on the internal wall of the elevating column; $\beta_{w}$ - the heat transfer's coefficient, which depends on the structure of the gas-and-oil flow in the well and also depends on the features of the flow at the near-surface layer of the channel's wall.

On the other hand the heat flow is defined by expression (18), is equal to the heat flow from the well's external wall into surrounding rock:

$$
Q=2 \pi R_{0} q_{w}=2 \pi R_{c} q, \quad q=\beta\left(T_{w}-T_{e x t}\right),
$$

where $T_{\text {ext }}$ is the geothermal temperature.

Solving last expression relatively $T_{w}$, we have:

$$
T_{w}=\frac{\beta_{w} R_{0} T+\beta R_{c} T_{e x t}}{\beta_{w} R_{0}+\beta R_{c}} .
$$

The second site is located between the section of beginning of the solid phase's formation and the well's head. On this site of the well where there are paraffin deposits, the intensity of heat exchange is described by expression:

$$
q_{w}=\beta_{w}\left(T-T_{\sigma}\right)
$$

where $T_{\sigma}$ is the temperature of the internal surface of the solid phase.

For the heat transfer's coefficient $\beta_{w}$ it is possible to write down expression:

$$
\beta_{w}=\gamma \frac{N u \cdot \lambda}{2 R}, \quad\left(\lambda=\alpha \lambda_{g}+(1-\alpha) \lambda_{l}, \quad N u=0,021 R e^{0,8} \stackrel{0,43}{\mathrm{Pr}}\right)
$$

where $\gamma$ - the correction factor to consider the structure of the flow, $\lambda_{i}(i=l, g)$ - heat conduction coefficient of the $i$ th phase.

At the mathematical formulation of the well's work with the IECP we will accept following assumptions: the characteristic size of the pump can be neglected in comparison with the length of the well $(L \ll H)$; used parameters at passage through the pump are testing jump.

The pressure jump it is possible to consider as function from the mass flow of the gas-liquid mixture and to approximate in the kind:

$$
\Delta p=A_{0}+A_{1} \cdot m+A_{2} \cdot m^{2},
$$

where $A_{0}, A_{1}, A_{2}$ - counted coefficients of a polynomial.

Change of temperature of the liquid-gas flow as the result of heat exchange with the IECP, taking into account thermal losses to surrounding rock, can be defined from the equation:

$$
\Delta T=\left(T_{1}+\frac{B_{1}}{\tilde{\beta}}\right)\left(\exp \left[\frac{L \tilde{\beta}}{m c}\right]-1\right),
$$

where $\tilde{\beta}=-\left(\beta_{d}+\beta\right), B_{1}=\beta_{d} T_{d}+\beta T_{\text {ext }}, T_{1}$ is the temperature of the gas-and-oil flow before the pump, $T_{d}$ - the temperature of the engine of the pump; $\beta_{d}$ - heat transfer's coefficient between the liquid-gas flow and the engine.

Change of mass concentrations of the light component in the liquid and gas phases at passage through pump can be defined on the basis of such parameters, as the pressure 
and the temperature of the two-phase flow:

$$
\begin{aligned}
& k_{l(L)}=\frac{p^{+}-p_{(M)}\left(T^{+}\right)}{G_{(L)}}, \quad k_{g(L)}=\left(1+\frac{R_{(L)} p^{+}}{R_{(M)}\left[p^{+}-p_{(M)}\left(T^{+}\right)\right]}\right)^{-1}, \\
& p_{(M)}\left(T^{+}\right)=p_{*} \exp \left(-\frac{T_{*}}{T^{+}}\right), \quad p^{+}=p_{1}+\Delta p, \quad T^{+}=T_{1}+\Delta T
\end{aligned}
$$

where $p_{1}$ is the pressure of the two-phase flow before IECP.

At the calculation of paraffin deposits' intensity on the inner walls of the channel we take into account that the mechanism of paraffin formation is the crystallization mechanism. Deposition of paraffin is starting in such sites of the well, where oil get in touch with the cold walls of the injection column. For each concentration of heavy high-molecular hydrocarbons in the gas-and-oil mixture the temperature of beginning the crystallization $T_{c}$ is existing. Below this temperature, falling of the hard phase from the flow is beginning.

For calculation of current position of border "gas-and-oil mixture - paraffin" it is possible to write down expression [5]:

$$
\begin{aligned}
& \frac{\partial \delta_{s}}{\partial t}=\frac{\beta_{s}\left(T_{w}-T_{c}\right)-\beta_{w}\left(T-T_{c}\right)}{\rho_{s}^{0}\left[L_{s}+\left(\beta_{w}-\beta_{s}\right) / K\right]}, \\
& \beta_{s}=\frac{\lambda_{s}}{R \ln \left(R / R_{0}\right),} \quad K=\frac{\rho_{l}^{0} D S h}{2 R}\left(\frac{\partial k_{l(H) e}}{\partial T_{\sigma}}\right)_{T_{c}}, \\
& k_{l(H) e}=k_{l(H) e}^{*} \exp \left(-T_{H}^{*} / T_{\sigma}\right), \quad T_{\sigma}=\frac{\beta_{w} T+K L_{s} T_{?}-\beta_{s} T_{w}}{\beta_{w}+K L_{s}-\beta_{s}},
\end{aligned}
$$

where $\rho_{s}^{0}$ and $\lambda_{S}$ are, accordingly, true density and heat conductivity coefficient of the solid phase; $D$ - diffusion coefficient; $L_{S}$ - the specific heat of the paraffin formation; $k_{l(H) e}$ is the equilibrium concentration of the heavy component; $k_{l(H) e}^{*}$ and $T_{H}^{*}$ are the empirical approximation parameters; $S h$ - Sherwood's number, $S h=\sqrt{2 R \cdot v_{l} / D}$.

\section{THE RESULTS OF THE NUMERICAL CALCULATIONS}

At carrying out of the numerical calculations the following parameter's values were used:

$m=1 \mathrm{~kg} / \mathrm{sec} ; m_{(L)}=0,018 \mathrm{~kg} / \mathrm{sec} ; R_{0}=0,0315 \mathrm{~m} ; R_{1}=0,0365 \mathrm{~m} ; R_{2}=0,084 \mathrm{~m} ;$ $R_{3}=0,089 \mathrm{~m} ; R_{c}=0,125 \mathrm{~m} ; H=2000 \mathrm{~m} ; H_{F}=200 \mathrm{~m} ; p_{b}=20 \mathrm{MPa} ; p_{f}=15 \mathrm{MPa} ;$ $T_{f}=328 \mathrm{~K} ; \varepsilon=1,5 \cdot 10^{-5} \mathrm{~m} ; \rho_{l}^{0}=850 \mathrm{~kg} / \mathrm{m}^{3} ; R_{(M)}=52 \mathrm{~m}^{2} /\left(\mathrm{sec}^{2} \mathrm{~K}\right) ; R_{(L)}=520$ $\mathrm{m}^{2} /\left(\sec ^{2} \mathrm{~K}\right) ; \lambda_{l}=0,13 \mathrm{kgm} /\left(\sec ^{3} \mathrm{~K}\right) ; c_{l}=2100 \mathrm{~m}^{2} /\left(\mathrm{sec}^{3} \mathrm{~K}\right) ; c_{g}=2500 \mathrm{~m}^{2} /\left(\sec ^{3} \mathrm{~K}\right) ;$ $L_{(M)}=2,3 \cdot 10^{5} \mathrm{~m}^{2} / \mathrm{sec}^{2} ; L_{(L)}=10^{4} \mathrm{~m}^{2} / \mathrm{sec}^{2}$,

$m_{(L)}=$ const - the mass flow of the light component. $p_{f}$ and $T_{f}$ the pressure and the temperature of the flow on the well's bottom. The temperature on the external border of the well is equaled to the geothermal temperature, in the annular space there is oil.

It is known, that on those sites of a well where the temperature of the elevating column's internal surface $T_{w}$ becomes below the temperature of the beginning of crystallization of paraffin $T_{c}$, for the short time the layer of paraffin deposits of a small thickness 
is formed. If not to accept any measures, there will be a gradual escalating of paraffin deposits until full overlapping of the well's cross section.
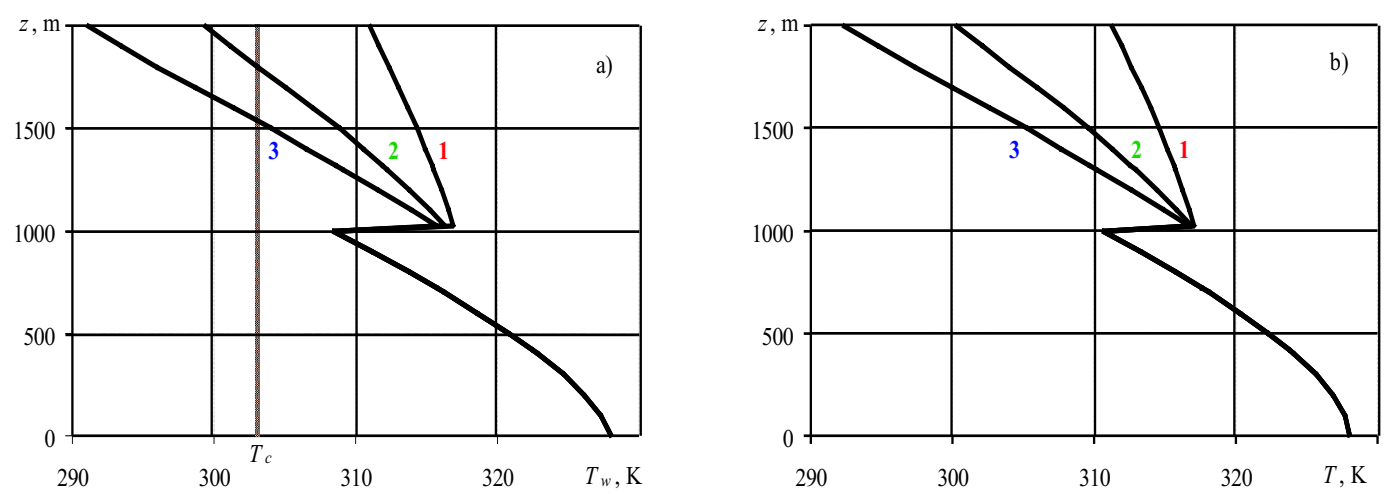

Fig. 1. Distributions on co-ordinate $z$ of the temperature of the elevating column's inner wall $T_{w}$ (a) and the average temperature of the flow $T$ (b) for the different fluids in the gap. Lines 1, 2 and 3 correspond to gas, oil and water. $T_{c}=303 \mathrm{~K}$, $z_{p}=1000 \mathrm{~m}, M_{p}=1 \mathrm{~kg} / \mathrm{s}$.
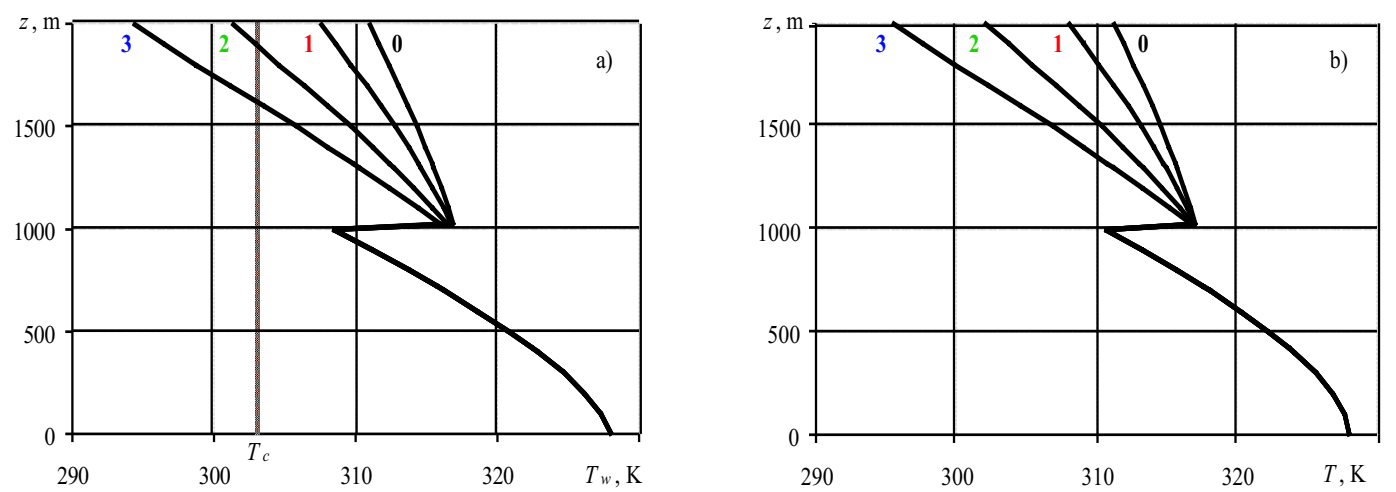

Fig. 2. Distributions on co-ordinate $z$ of the inner wall's temperature $T_{w}$ (a) and the average temperature of the flow $T$ (b) for the case of convective gas flow in gap. Lines 1, 2, 3 correspond to the $\mathrm{Gr}=10^{5}, 10^{6}, 10^{7}$; the line 0 - $\mathrm{Gr}=0$.

Therefore it is necessary to take preventive methods of struggle against paraffin deposits in a well. As one of such methods the complex of actions for heat transfer's decrease through system of pipes of a well can serve. On the Fig. 1 distributions of the well's temperatures depending on fluids in the gap are presented. If the gap of a well is filled by fluid with smaller factor heat conductivity (gas) the temperature mode in the well appreciably improves. If gas in the gap is in the condition of thermogravitational convection at intensity reduction of convective flow the temperature in the chink's elevating column is raising (Fig. 2). For heat transfer reduction through system of well's pipes it is possible to use the pipes from heat-insulating material (Fig. 3). 

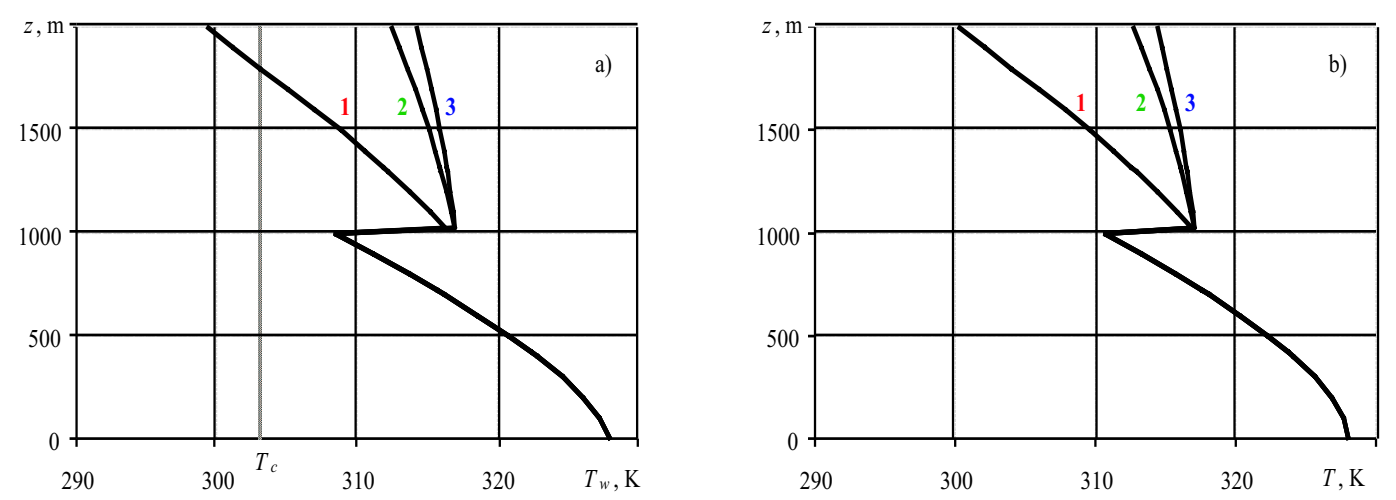

Fig. 3. Distributions of the inner wall's temperature $T_{w}$ (a) and the average temperature of the flow $T(\mathrm{~b})$ on co-ordinate $z$. Lines 1,2 and 3 correspond to the thickness of the heat-insulating material (polyurethane foam, $\lambda=0,0067 \mathrm{Watt} /(\mathrm{m}$ $\mathrm{K})$ ) on the external border of the elevating column $\mathrm{dh}=0,5,10 \mathrm{~mm}$; surface gap is filled by oil.
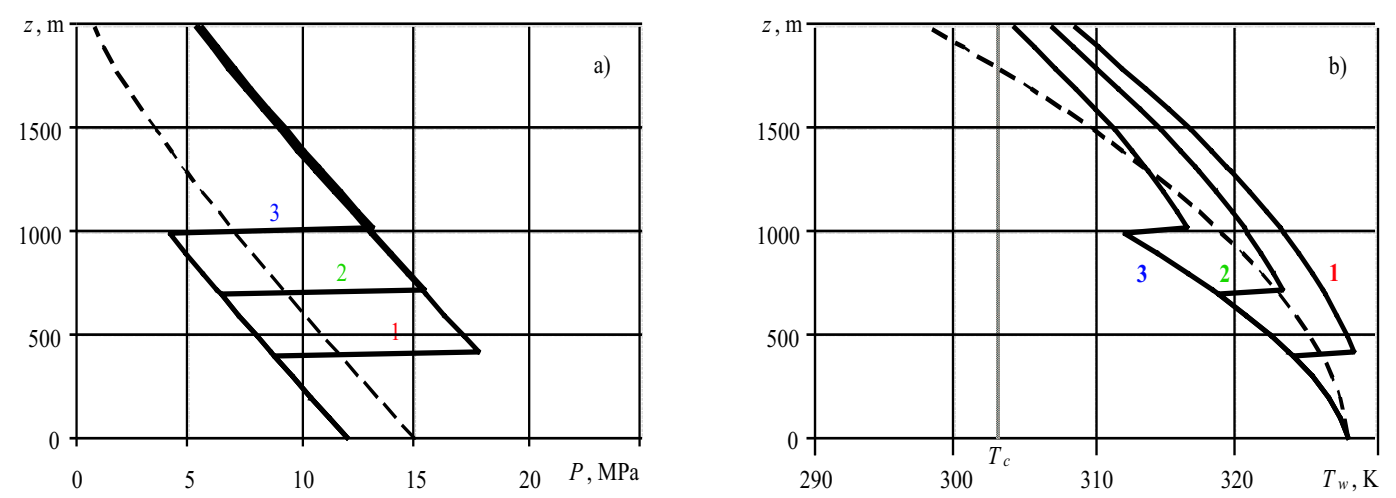

Fig. 4. Distributions of the flow's pressure (a) and the inner wall's temperature (b) on co-ordinate $z$. Lines 1,2 and 3 correspond to the depth of pump's setting $z_{p}=400,700$ and $1000 \mathrm{~m}, M_{p}=1,6 \mathrm{~kg} / \mathrm{s}$; dashed line - no pump, the elevating column is lowered to the well bottom.

On the Fig. 4 and Fig. 5 distributions of the flow's pressure and the temperature of the elevating column's internal wall depending on location the IECP and from the pump's productivity $M_{p}$ are presented. The Fig. 6 illustrates the dependence of the inner wall's temperature of the elevating column at the well head from the pump location. It is visible, that at arrangement of the pump above 1100 meters, the elevating column's temperature $T_{w}$ becomes below temperature of the beginning paraffin's crystallization $T_{c}$, therefore formation of paraffin deposits is possible. Accordingly the pump use essentially changes hydrodynamic and temperature conditions in the well. 

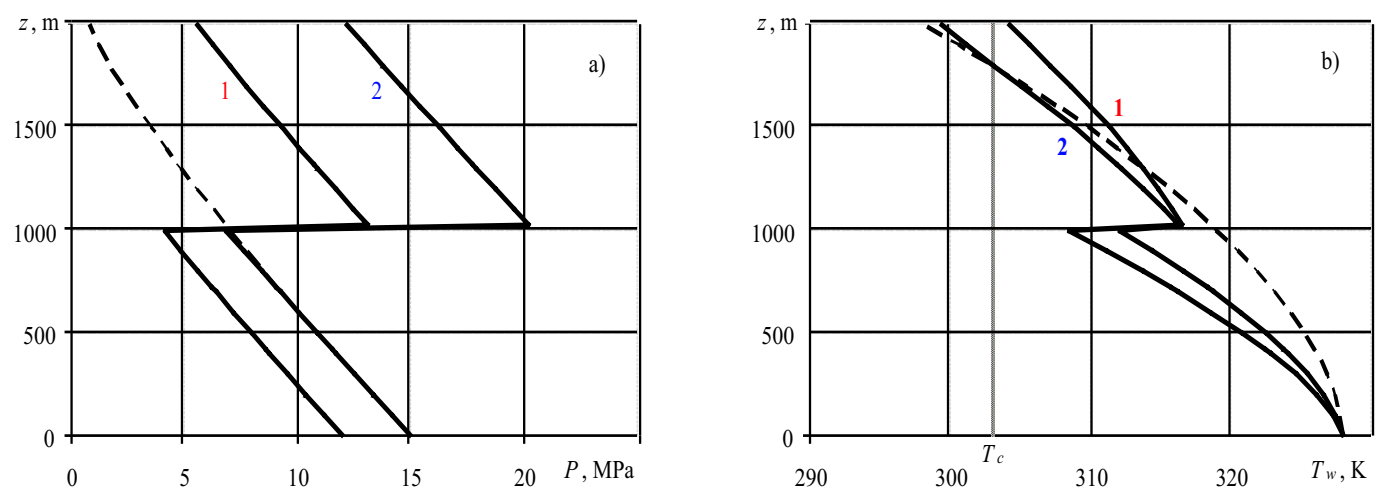

Fig. 5. Distributions of the flow's pressure (a) and the inner wall's temperature (b) on co-ordinate $z$. Lines 1 and 2 correspond to metering characteristic of the pump $M_{p}=1,6$ and $1 \mathrm{~kg} / \mathrm{s}, z_{p}=1000 \mathrm{~m}$; dashed line - no pump, the elevating column is lowered to the well bottom.

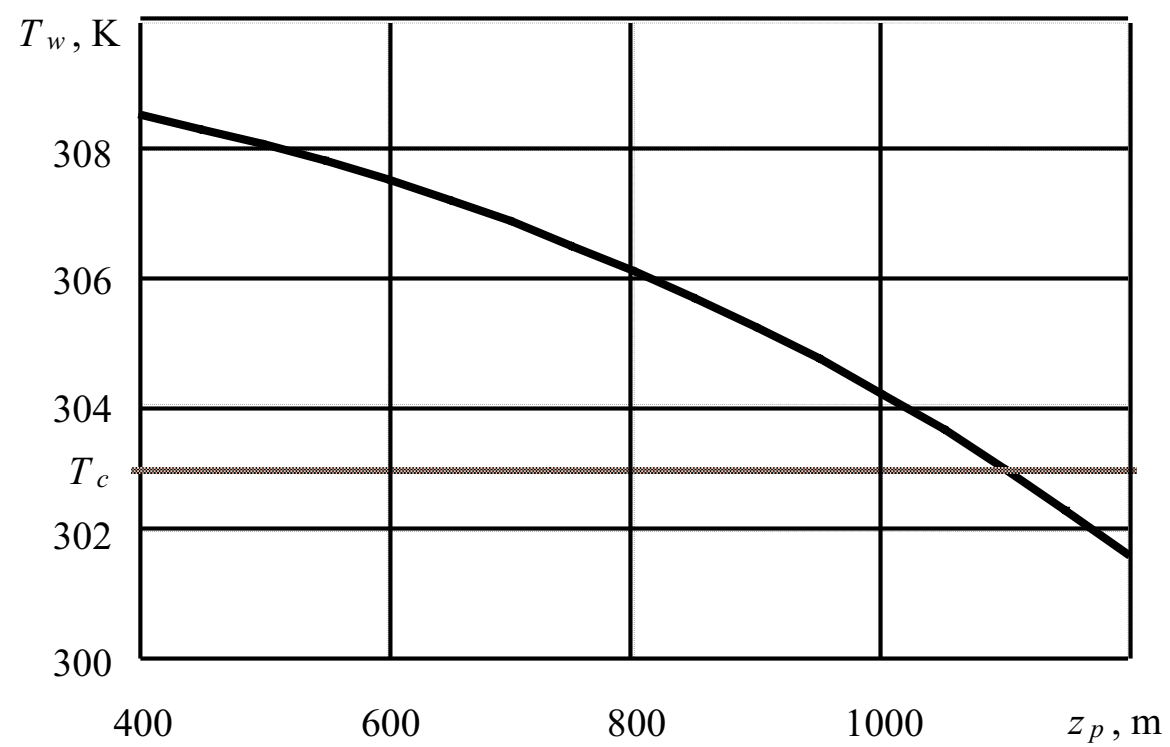

Fig. 6. Dependence of the inner wall's temperature of the elevating column at the well head from the pump location; $M_{p}=1,6 \mathrm{~kg} / \mathrm{s}$.

\section{CONCLUSION}

The mathematical model of an upward gas-and-oil flow consisting of three interconnected blocks is developed:

- Hydrodynamics and thermal physics of the gas-and-oil flow in a well's elevating column with an IECP;

- Growth of paraffin deposits on the elevating column's internal walls; 
- Thermal interaction of the gas-and-oil flow in the well with surrounding rock.

It is shown, that at the using of an installation of electrical centrifugal pumps and variation of its metering characteristic, depth of its setting, it is possible to achieve the optimum temperature mode on all the well's height and to prevent by it formation of paraffin deposits.

Work is executed at financial support of the Russian fund of basic researches (the project 08-01-90300).

\section{REFERENCES}

[1] V.Sh. Shagapov, N.G. Musakaev, N.S. Khabeev, S.S. Bailey, Mathematical modelling of two - phase flow in a vertical well considering paraffin deposits and external heat exchange, International Journal of Heat and Mass Transfer, 47(4) (2004) 843 - 851.

[2] R.I. Nigmatulin, Dynamics of Multiphase Media, Hemisphere Publ. Corp, NewYork, (1991).

[3] D. Chisholm, Two-phase flow in pipelines and heat exchangers, Publ. Longmen Group Ltd, London, (1983).

[4] J. Burger, P. Sourieau, M. Combarnous, Recuperation assistee du petrole les methodes thermiques, Paris, (1988).

[5] V.Sh. Shagapov and N.G. Musakaev, Modeling of Paraffin Deposition in Flow of a Gas-Oil Mixture in Tubes, Journal of Engineering Physics and Thermophysics, 72(4) (1999) 744 747.

Received April 06, 2010

\section{NGHIÊN CƯU LÝ THUYẾT DÒNG CHẢY ĐI LÊN DẦU - KHÍ TRONG GIẾNG ĐỨNG CÓ CHUYỂN PHA}

Bằng phương pháp cơ học các hệ nhiều pha, đã trình bày mô hình toán học dòng chảy dầu - khí trong giếng đứng. Mô hình có xét đến chuyển pha, lắng đọng paraffin trên thành giếng, ảnh hưởng sự có mặt của bơm điện lên các tham số dòng chảy hỗn hợp, cũng như sự trao đổi nhiệt của giếng với môi trường xung quanh.

Đã chỉ ra rằng, có thể đạt được điều kiện tối ưu về nhiệt độ dọc theo thân giếng nhằm ngăn ngừa khả năng kết tinh và lắng đọng paraffin thông qua sự thay đổi các tham số liên quan đến máy bơm điện, độ sâu đặt bơm. 